\title{
Perfil socioeconômico dos cuidadores de crianças com hidrocefalia
}

\author{
Débora Moura da Paixão Oliveira1 , Carlos Umberto Pereira², Záira Moura da Paixão Freitas ${ }^{3}$ \\ Universidade Federal de Sergipe (UFS), Aracaju, SE, Brasil.
}

\section{RESUMO}

Objetivo: Caracterizar cuidadores de crianças com hidrocefalia em relação ao perfil socioeconômico. Método: Estudo descritivo, quantitativo, utilizando a técnica de entrevista padronizada com cuidadores atendidos no ambulatório de um hospital universitário entre novembro de 2007 e agosto de 2008. Foram coletados os dados socioeconômicos como idade, gênero, parentesco, procedência, escolaridade, situação conjugal, moradia, ocupação, renda familiar, número de moradores no domicilio. Para análise estatística, foram utilizados os testes de qui-quadrado de Pearson, exato de Fisher e McNemar. Resultados: A amostra foi composta por 54 cuidadores procedentes do interior do estado de Sergipe, Bahia e Alagoas, com média de idade de $27,3 \pm 7$ anos e predomínio absoluto do gênero feminino. Observou-se um efeito significativo $(p=0,00004)$ sobre o número das que deixaram de trabalhar para ser cuidadoras. Vinte e três (42,6\%) cursaram até oito anos de estudo. Trinta cuidadoras $(55,6 \%)$ possuem renda familiar de um salário-mínimo. Conclusão: Os achados sugerem que cuidadoras de crianças com hidrocefalia são donas de casa sem remuneração, com baixo grau de instrução e pouca orientação profissional. Deixaram de trabalhar para ser cuidadoras e possuem recursos financeiros reduzidos.

\section{PALAVRAS-CHAVE}

Criança, cuidadores, familia, hidrocefalia.

\begin{abstract}
Socioeconomic profile of familial caregiver's hydrocephalus

Objective: To characterize the socioeconomic profile of caregivers of hydrocephalus patients. Method: Descriptive and quantitative study using the interview technique. The survey was performed with caregivers at a University Hospital between November 2007 and August 2008. We collected socioeconomics data as age, gender, relationship, origin, scholarship, marital status, residence, job, familial income, number of people at home. For statistical analyses was utilized chi-square, Pearson, Fisher exact and McNemar tests. Results: Fifty-four caregivers answered the interview, 38 (70.4\%) come from rural zone the state of Sergipe, Bahia and Alagoas with mean age $27.3 \pm 7$ years, and absolute predominance of females. There was a positive correlation on the number that they had left to work to be caregivers $(p=0.00004)$. Twenty-three (42.6\%) studied only eight years. Thirty caregivers (55.6\%) have lower family income a month. Conclusion: The caregivers of hydrocephalus patients are mothers without remuneration with have a poor education level and they received little professional information. They had left to work to be caregivers and reduced financial resources.
\end{abstract}

\section{KEYWORDS}

Child, caregivers, family, hydrocephalus.

\section{Introdução}

A hidrocefalia é uma condição em que a principal característica é o acúmulo excessivo de líquido nos ventrículos cerebrais. ${ }^{17,31} \mathrm{O}$ tratamento da hidrocefalia consiste em reduzir a quantidade de líquido no cérebro, a fim de diminuir a pressão intracraniana. ${ }^{31} \mathrm{~A}$ criança com hidrocefalia passa por diversos procedimentos que diferem da rotina de uma criança saudável. ${ }^{1,20}$ Esses procedimentos acabam fazendo parte da vida desses indivíduos e de suas famílias, uma vez que, em situação de doenças crônicas, os cuidados nunca cessam. ${ }^{8,25}$

Os cuidados necessários dependem da idade da criança, da causa da hidrocefalia e do tipo de intervenção médica a ser realizada. No domicílio, a criança recebe cuidados de pessoas com algum grau de parentesco e

\footnotetext{
1. Secretaria Municipal de Saúde de Aracaju, Universidade Federal de Sergipe (UFS), Aracaju, SE, Brasil.

2. Departamento de Medicina da UFS, Aracaju, SE, Brasil.

3. Maternidade Nossa Senhora de Lourdes, UFS, Aracaju, SE, Brasil.
}

Extraído da dissertação Caracterização do cuidador familiar em relação ao conhecimento sobre hidrocefalia, apresentada ao Núcleo de Pós-Graduação em Medicina e Saúde da Universidade Federal de Sergipe. 
proximidade física ou afetiva, geralmente do gênero feminino, que se dedicam, sem remuneração, a empreender cuidados na vida cotidiana para aquelas que inspiram cuidados especiais, supervisão e assistência. ${ }^{13,16,29}$

O cuidador realiza práticas de cuidado dentro da sua realidade, visando amenizar sintomas, oferecer alimentação adequada, cuidar do vestuário e aplicar medidas de conforto visando à prevenção de complicações pós-operatórias. ${ }^{1,23}$

Entretanto, há de se considerar que a hidrocefalia envolve questões além da própria enfermidade, envolve a estrutura e o aspecto socioeconômico familiar. ${ }^{15,30}$ O tratamento da criança com hidrocefalia exige da família capacidade cognitiva dos cuidadores para realizar cuidados técnicos, muitas vezes complexos, visitas regulares a serviços de saúde e, em algumas situações, despesas extras não cobertas pelos benefícios sociais. ${ }^{27}$

Vários autores demonstraram o efeito do status socioeconômico da família na saúde da criança, ${ }^{19,21,26}$ verificando-se que uma renda mais baixa foi associada com maior necessidade de cuidados médicos e piores resultados sociais e cognitivos.

Kulkarni et al. ${ }^{15}$ identificaram que as disparidades socioeconômicas permanecem como importantes causas determinantes de maior qualidade de vida em crianças com hidrocefalia. Os autores perceberam que crianças de famílias com maior renda têm melhor acesso ao cuidado e acreditam que o impacto de fatores socioeconômicos da hidrocefalia também esteja relacionado a diferentes tipos de acesso ao cuidado.

Este estudo teve como objetivo descrever o perfil socioeconômico de cuidadores de crianças com hidrocefalia em uma capital do nordeste do país, uma vez que pensar no bem-estar dos cuidadores é interferir indiretamente na qualidade de vida das crianças com hidrocefalia.

\section{Casuística e método}

Foi realizado um estudo descritivo, por meio de uma abordagem quantitativa, com cuidadores familiares de crianças com hidrocefalia.

A população-alvo foi constituída por 54 cuidadores de crianças com hidrocefalia escolhidas aleatoriamente segundo a sua presença para consulta no Ambulatório de Neurocirurgia da Universidade Federal de Sergipe, na cidade de Aracaju. A amostra foi acidental, não probabilística.

Neste estudo foi considerado como cuidador principal o cuidador exclusivamente familiar, não remunerado, responsável pelo cuidado domiciliar, e que permanece a maior parte do tempo com a criança.
Para a coleta de dados, utilizou-se um formulário de perguntas fechadas para entrevista padronizada, englobando variáveis definidas para o objetivo da pesquisa. O instrumento foi testado em um estudo piloto com dez cuidadores. Verificou-se não haver problemas com as questões. Os resultados demonstraram ótima compreensão e entendimento com um tempo médio de 40 minutos para seu preenchimento.

Foram coletados os dados socioeconômicos como idade, gênero, parentesco, procedência, escolaridade, situação conjugal, moradia, ocupação, renda familiar, número de moradores no domicílio.

A coleta de dados procedeu-se no período de novembro de 2007 a agosto de 2008 após a aprovação do projeto de pesquisa pelo Comitê de Ética em Pesquisa com $\mathrm{n}^{\circ}$ 0126.0.107.000-07. Para análise estatística, foram utilizados os testes qui-quadrado de Pearson, exato de Fisher e McNemar com a ajuda do programa SPSS (Statistical Package for Social Sciences) versão 15.0.

\section{Resultados}

Em relação ao gênero, revelou-se a predominância do gênero feminino $\mathrm{N}=54(100 \%)$, com idade variando de 18 anos a 52 anos (média 27,3 \pm 7 anos).

A análise do grau de parentesco do cuidador demonstrou predominância significativa de mães $(\mathrm{p}<$ 0,0001), sendo $53(98,1 \%)$ mãe biológica e uma avó $(1,9 \%)$.

Em relação à procedência dos cuidadores, observou-se que $38(70,4 \%)$ procederam do interior, não só do estado de Sergipe, mas dos estados da Bahia e de Alagoas $(\mathrm{p}=0,003)$.

No que se refere ao grau de instrução, $13(24,1 \%)$ cursaram menos de quatro anos de estudo, 23 (42,6\%), entre quatro e oito anos e $18(33,3 \%)$, até 11 anos de estudo.

A análise da situação conjugal verificou que 44 cuidadoras $(81,5 \%)$ vivem com um companheiro ( $\mathrm{p}<$ $0,0001)$ e outros filhos e $10(18,5 \%)$ cuidadoras vivem sem o pai da criança $(18,5 \%)$.

Quanto à moradia, apenas $11(20,3 \%)$ possuem casa própria, sendo a maior proporção de cuidadores ( $43 \%$ - 79,7\%) residentes em casa alugada ou de parentes.

Em relação à ocupação, observou-se que antes do nascimento da criança 27 cuidadoras $(50 \%)$ possuíam atividade remunerada; após o nascimento da criança, apenas nove (16,7\%) mantiveram seus empregos, ou seja, $18(66,6 \%)$ cuidadoras afastaram-se do trabalho para dedicar-se aos cuidados. Verificou-se um efeito significativo (McNemar $\mathrm{p}=0,00004 ; \mathrm{N}=54)$ sobre o número das que deixaram de trabalhar para ser cuidadoras (Figura 1). 


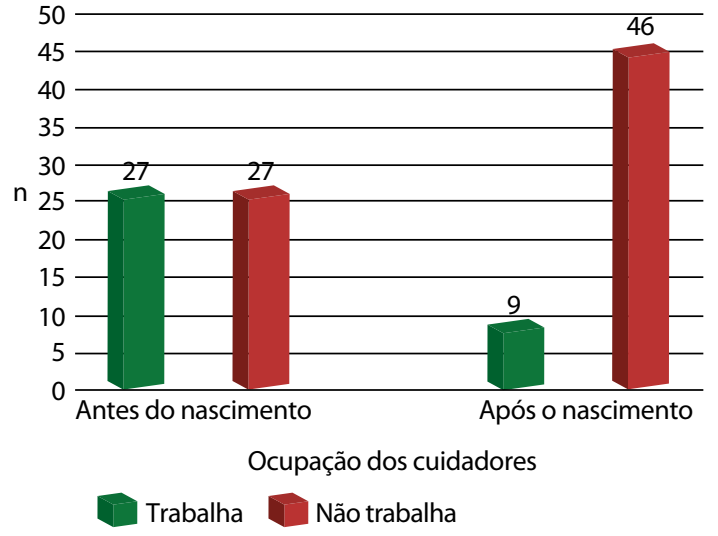

Figura 1 - Número de cuidadoras que deixaram de trabalhar para dedicar-se ao cuidado.

Em relação à renda familiar, 30 cuidadoras $(55,6 \%)$ têm renda de um salário-mínimo, apenas oito (14,8\%) têm renda familiar de até dois salários-mínimos e 16 $(29,6 \%)$ vivem com menos de um salário-mínimo.

A análise do número de pessoas existente por domicílio mostrou que 34 cuidadoras $(63 \%)$ convivem com mais de quatro pessoas ( $\mathrm{p}<0,0001), 14(25,9 \%)$ conviviam com três pessoas e seis $(11,1 \%)$ vivem com apenas duas pessoas no domicílio.

\section{Discussão}

A hidrocefalia envolve questões não só a respeito da própria enfermidade, como relativas ao aspecto socioeconômico familiar. A caracterização dos cuidadores deste estudo em relação ao gênero revelou a predominância do gênero feminino. Nossos achados estão de acordo com a literatura, que destaca o papel da mulher como cuidadora. É comum afirmar que compete à mulher a tarefa de cuidar da casa, dos filhos ou dos idosos e, apesar da inserção da mulher no mercado de trabalho, ela ainda mantém características de prover cuidados. ${ }^{10,12,16}$

A média de idade dos cuidadores encontrada neste estudo foi de 27,3 anos, o que se aproxima da média etária encontrada em outros estudos. Um estudo desenvolvido por Ludueña e Prada ${ }^{18}$ descreveu a prevalência de mielomeningocele em filhos de mães jovens. Uma investigação com cuidadores, realizada em 1999, mostrou que a idade das mães variou entre 15 e 43 anos, sendo metade da amostra com idade entre 19 e 29 anos. ${ }^{4}$ Essas mães acabam participando como cuidadores muito cedo, uma característica importante também observada neste estudo.
Em relação ao parentesco, percebeu-se predominância de mães cuidadoras. Estudos coincidem em afirmar que a própria mãe acredita que ninguém está à altura de cuidar de seu filho e protegê-lo. ${ }^{10,22}$ Essa determinação está ligada a fatores como a proximidade afetiva com a pessoa que requer o cuidado e ao vínculo consanguíneo, bem como está inscrita socialmente no papel de mãe. ${ }^{10}$ Esse papel é visto como natural, no entanto, quanto maior o vínculo familiar, maior será a sobrecarga física e emocional envolvida no processo de saúde e doença. ${ }^{5}$

A procedência do cuidador foi pesquisada com o objetivo de observar possíveis tendências migratórias para o município de Aracaju, fato esse confirmado com um número expressivo de cuidadores procedentes de outros municípios. Observa-se uma correspondência entre as características das cuidadoras aqui analisadas com as de outros estudos. Os autores investigaram o local de residência das famílias, constatando que grande parte delas residia em municípios do interior do estado. ${ }^{3}$ Em São Paulo, uma investigação com crianças com hidrocefalia demonstrou que $62 \%$ de moradores eram procedentes do interior do estado e $15 \%$, de outros estados do país. ${ }^{14}$

Considerando que o local da realização da pesquisa é referência para todo o estado de Sergipe, os resultados permitem supor que moradores de outros municípios e estados vizinhos deslocam-se para a capital em busca de tratamento especializado.

Observou-se um bom índice de alfabetização entre as entrevistadas, porém a escolaridade média localiza-se em maior proporção entre cuidadoras que cursaram quatro a oito anos de estudo. Os resultados encontrados por outros autores no Brasil também demonstram um padrão de escolaridade mínima, semelhante a esta casuística; as mulheres que assumem o cuidado cursaram apenas o ensino fundamental. ${ }^{12,19,22,28}$ A baixa escolaridade observada no estudo pode ser relacionada à instituição local de pesquisa, que atende às classes sociais menos favorecidas usuárias do Sistema Único de Saúde.

Porém, é preciso considerar a capacidade da família para cuidar de seus membros, uma vez que a educação em saúde está relacionada à capacidade de aprendizagem das pessoas..$^{24}$ As informações só são assimiladas a partir do momento em que haja capacidade cognitiva da família, caso contrário não haverá aprendizado nem compreensão sobre a importância do tratamento. ${ }^{7}$ Em 2008, no Canadá, uma investigação demonstrou que nenhum familiar com nível universitário foi determinante para menor qualidade de vida em crianças com hidrocefalia. ${ }^{15}$

O estudo mostrou que as cuidadoras viviam em casas alugadas. A falta de moradia própria é um problema observado na literatura. Gaiva et al. ${ }^{9}$ perceberam que cuidadores de crianças com espinha bífida também viviam em casas alugadas ou cedidas e poucos 
possuíam casa própria, o que pode estar relacionado a pouco recurso financeiro.

Cuidar de enfermo também envolve dimensões financeiras, assim sendo foram analisados os cuidadores em relação a sua ocupação e renda. Esse resultado concorda com outros estudos, em que as mães cuidadoras eram donas de casa sem remuneração e deixaram seus empregos para dedicar-se ao cuidado. As cuidadoras ocupavam-se com serviços domésticos em seus próprios domicílios; algumas relataram não poder assumir uma ocupação remunerada extradomiciliar em virtude dos cuidados dispensados ao doente. ${ }^{4,28}$

Um estudo desenvolvido em 2006 no Brasil mostrou que o impacto causado pelo desemprego afeta um número bem maior de pessoas, uma vez que o desempregado integra uma família que, consequentemente, será afetada. Esse impacto dependerá da posição que o trabalhador ocupa na família e dos recursos de que dispõe. ${ }^{2} \mathrm{O}$ estudo também mostrou que o desemprego concentra-se nos segmentos mais jovens e entre as mulheres.

A dificuldade financeira é um problema importante vivenciado pelas famílias de pacientes crônicos; pessoas de classes sociais mais baixas são mais frequentemente acometidas por essas doenças. ${ }^{3,18,22}$ Uma investigação em 150 crianças com defeitos do tubo neural na cidade de São Paulo registrou um nível socioeconômico baixo, com altos contingentes de origem nordestina. ${ }^{19}$

Esses resultados podem indicar que a incidência de hidrocefalia é maior em famílias de menor renda.

Mães de crianças deficientes têm taxas de empregos menores que mães de filhos saudáveis, com isso apresentam em média recursos mais reduzidos que famílias em geral, uma vez que muitas vezes essas famílias enfrentam custos extras. ${ }^{27}$

O tamanho da família também está associado à sua situação socioeconômica. Esses dados confirmam a situação abaixo da pobreza em que vivem essas famílias. A falta de recursos e estrutura familiar, além de contribuir para a deterioração das condições de vida da família, diminui sua autoestima, interferindo na qualidade das relações familiares e, consequentemente, afetando a saúde da criança. ${ }^{11,23,30}$

Outros estudos verificaram que a saúde da criança com hidrocefalia está associada não só a fatores relacionados ao tratamento, complicações, sequelas e acesso ao cuidado, mas também a fatores socioeconômicos como educação dos pais, estrutura, emprego e renda familiar. ${ }^{6,23,30}$

A maioria das cuidadoras vive em famílias nucleares, um achado importante, uma vez que a literatura menciona que a estrutura familiar de famílias nucleares pai, mãe e filho é uma variável de grande influência na saúde da criança. ${ }^{23,30}$ Existe uma clara associação entre a unidade familiar e a qualidade de vida dessas crianças. No entanto, nossos achados mostraram também que dez cuidadoras não possuíam famílias nucleares, já que vivem sem o pai da criança. Esse fato pode ser inerente ao efeito adverso da pior função familiar, sendo igualmente possível dizer que o funcionamento da família se deteriora secundariamente nas famílias de crianças com saúde mais pobre. ${ }^{15}$

Porém, o fato de conviverem com o companheiro e outros filhos pode identificar que muitas cuidadoras precisam dividir o seu tempo entre os afazeres domésticos e o cuidado com a família, o que pode gerar a sobrecarga do cuidador. ${ }^{5}$

\section{Conclusão}

Os resultados obtidos permitiram concluir que os cuidadores de crianças com hidrocefalia são jovens mães, procedentes do interior, com baixo grau de instrução. São donas de casa sem remuneração, uma vez que deixaram de trabalhar para ser cuidadoras, possuem recursos financeiros reduzidos e não possuem moradia própria. Vivem com um companheiro e mais de quatro pessoas no domicílio, demonstrando a situação de pobreza em que vivem essas famílias.

Os autores declaram inexistência de conflito de interesses na realização deste trabalho.

\section{Referências}

1. Andrade MB, Dupas G, Wernet M. Convivendo com a criança com hidrocefalia: experiência da família. Cienc Cuid Saude. 2009;8(3):436-43.

2. Borges A. Impactos do desemprego e da precarização sobre famílias metropolitanas. Rev Bras Estud Popul. 2006;23(2):205-22.

3. Bornstein MH, Hahn CS, Suwalsky JTD, Haynes OM. The Hollingshead four-factor index of social status and the socioeconomic index of occupations. In: Bornstein MH, Bradley $\mathrm{RH}$, editors. Socioeconomic status, parenting, and child development. Mahwah, New Jersey: Lawrence Erlbaum; 2003. p. 29-82.

4. Cáceres FM, Uscátegui AM, Rojas JD, Becerra CH, Díaz LA. Incidencia de las malformaciones congénitas. Registro durante un año de vigilancia activa no selectiva en el Hospital Universitario Ramón Gonzalez Valencia. Medunab. 1999;2(6):109-14.

5. Camargos ACR, Lacerda TTB, Viana SO, Pinto LRA, Fonseca MLS. Avaliação da sobrecarga do cuidador de crianças com paralisia cerebral através da escala Burden Interview. Rev Bras Saúde Matern Infant. 2009;9(1):31-7.

6. Chen E, Martin AD, Matthews KA. Socioeconomic status and health: do gradients differ within childhood and adolescence? Soc Sci Med. 2006;62(9):2161-70.

7. Damas KCA, Munari DB, Siqueira KM. Cuidando do cuidador: reflexões sobre o aprendizado dessa habilidade. Rev Eletrônica Enferm. 2004;6(2):272-8. 
8. Fisher HR. The needs of parents with chronically sick children: a literature review. J Adv Nurs. 200;36(4):600-7.

9. Gaiva MA, Neves AQ, Siqueira FM. O cuidado da criança com espinha bífida pela família no domicílio. Esc Anna Nery. 2009;13(4):717-25.

10. Gallo AM, Hadley EK, Angst DB, Knafl KA, Smith CA. Parents' concerns about issues related to their children's genetic conditions. J Spec Pediatr Nurs. 2008;13(1):4-14.

11. Gonzáles EMT, Munõz MCL, Guitiérrez SC, Palafox MAG. Factores psicosociales asociados al desarrollo de niños con mielomeningocele. Salud Mental. 2002;25(6):44-52.

12. Grippo ML, Fracolli LA. [Evaluation of an educational booklet about childcare promotion from the family's perception regarding health and citizenship]. Rev Esc Enferm USP. 2008;42(3):430-6.

13. Hunt CK. Concepts in caregiver research. J Nurs Scholarsh. 2003;35(1):27-32.

14. Jucá CEB, Lins Neto A, Oliveira RS, Machado HR. Tratamento de hidrocefalia com derivação ventrículoperitoneal: análise de 150 casos consecutivos no Hospital das Clínicas de Ribeirão Preto. Acta Cir Bras. 2002;7(Supl 3):59-63.

15. Kulkarni AV, Cochrane DD, McNeely PD, Shams I. Medical, social, and economic factors associated with health-related quality of life in Canadian children with hydrocephalus. J Pediatr. 2008;153(5):689-95.

16. Lackey NR, Gates MF. Adults' recollections of their experiences as young caregivers of family members with chronic physical illnesses. J Adv Nurs. 2001;34(3):320-8.

17. Lifshutz JI, Johnson WD. History of hydrocephalus and its treatments. Neurosurg Focus. 2001;11(2):E1.

18. Ludueña MP, Prada EMG. Características clínicas de recién nacidos internados por mielomeningocele en el Hospital del Niños "Dr. Ovidio Aliaga Uría" 1993-2002. Rev Soc Bol Ped. 2003;42(3):160-5.

19. Misisian APC. Defeitos de fechamento do tubo neural: estudo das condições socioeconômicas e da recorrência em uma amostra de pacientes da cidade de São Paulo [dissertação]. São Paulo: Escola Paulista de Medicina, Universidade Federal de São Paulo; 2000.

20. Oliveira DMP. Assistência de enfermagem à criança com hidrocefalia. In: Pereira CU. Neurocirurgia pediátrica. Rio de Janeiro: Revinter; 2000. p. 57-60.
21. Olson LM, Tang SF, Newacheck PW. Children in the United States with discontinuous health insurance coverage. N Engl J Med. 2005;353(4):382-91.

22. Pedro KS, Marcon SS. Perfil e vivência dos cuidadores informais de doentes crônicos assistidos pelo NEPAAF Núcleo de estudos, pesquisa, assistência e apoio à família. Online Braz J Nurs. 2007;6. Disponível em: <http://www. objnursing.uff.br>. Acesso em: 15 abril 2007.

23. Ray LD. Parenting and childhood chronicity: making visible the invisible work. J Pediatr Nurs. 2002;17(6):424-38.

24. Resta DG, Budó MLD. A cultura e as formas de cuidar em família na visão de pacientes e cuidadores domiciliares. Acta Sci Health Sci. 2004;26(1):53-60.

25. Rothman AA, Wagner EH. Chronic illness management: what is the role of primary care? Ann Intern Med. 200;138(3):256-61.

26. Silver EJ, Stein RE. Access to care, unmet health needs, and poverty status among children with and without chronic conditions. Ambul Pediatr. 2001;1(6):314-20.

27. Sloper P. Models of service support for parents of disabled children. What do we know? What do we need to know? Child Care Health Dev. 1999;25(2):85-99.

28. Souza CS, Alves AMA. As representações e expectativas de mães frente ao diagnóstico de hidrocefalia congênita de seus filhos. Rev Pesq (Rio de Janeiro). 2004;8(1/2):77-86.

29. Souza LM, Wegner W, Gorini MIPC. Educação em saúde: uma estratégia de cuidado ao cuidador leigo. Rev Latinoam Enfermagem. 2007;15(2):6-9.

30. Stevens GD. Gradients in the health status and developmental risks of young children: the combined influences of multiple social risk factors. Matern Child Health J. 2006;10(2):187-99.

31. Vannemreddy P, Nourbakhsh A, Willis B, Guthikonda B. Congenital Chiari malformations. Neurol India. 2010;58(1):6-14 .

\section{Endereço para correspondência}

Débora Moura da Paixão Oliveira

Av. Augusto Maynard, 245, ap. 404, São José

49015-380 - Aracaju, SE, Brasil

Telefone: (79) 9987-3209

E-mail: debora_aju@yahoo.com.br 